\title{
Scopolamine infused into perirhinal cortex improves object recognition memory by blocking the acquisition of interfering object information
}

\author{
Boyer D. Winters, ${ }^{1,3}$ Susan J. Bartko, ${ }^{1}$ Lisa M. Saksida, ${ }^{1,2}$ and Timothy J. Bussey ${ }^{1,2}$ \\ ${ }^{1}$ Department of Experimental Psychology, University of Cambridge, Cambridge CB2 3EB, United Kingdom; ${ }^{2}$ The MRC and \\ Wellcome Trust Behavioural and Clinical Neuroscience Institute, University of Cambridge, Cambridge CB2 3EB, United Kingdom
}

\begin{abstract}
In a previous study, we reported apparently paradoxical facilitation of object recognition memory following infusions of the cholinergic muscarinic receptor antagonist scopolamine into the perirhinal cortex (PRh) of rats. We attributed these effects to the blockade by scopolamine of the acquisition of interfering information. The present study tested this possibility directly by modifying the spontaneous object recognition memory task to allow the presentation of a potentially interfering object either before the sample phase or in the retention delay between the sample and choice phases. Presentation of an object between the sample and choice phases disrupted subsequent recognition of the sample object (retroactive interference), and intra-PRh infusions of scopolamine prior to the presentation of the irrelevant object prevented this retroactive interference effect. Moreover, presentation of an irrelevant object prior to the sample phase interfered proactively with sample object recognition, and intra-PRh infusions of scopolamine prior to the presentation of the pre-sample object prevented this proactive interference effect. These results suggest that blocking muscarinic cholinergic receptors in PRh can disrupt the acquisition of potentially interfering object information, thereby facilitating object recognition memory. This finding provides further, strong evidence that acetylcholine is important for the acquisition of object information in PRh.
\end{abstract}

The neurotransmitter acetylcholine (ACh) has long been thought to play an important role in learning and memory processes (Hasselmo and Bower 1993; Everitt and Robbins 1997; Bartus 2000). Indeed, this role has been highlighted by research using object recognition tasks to study mammalian declarative memory. Past findings have indicated that systemic administration of the muscarinic cholinergic receptor antagonist scopolamine before sample object exposure disrupts subsequent recognition of that same object after a delay (Aigner and Mishkin 1986; Huston and Aggleton 1987; Ennaceur and Meliani 1992; Dodart et al. 1997). One temporal lobe structure that is indispensable to the performance of delayed object recognition memory tasks is the perirhinal cortex (PRh) (Meunier et al. 1993; Buffalo et al. 1998; Murray and Bussey 1999; Winters et al. 2004). Accordingly, recent research has shown that permanent cholinergic denervation of PRh using immunotoxins impairs visual recognition memory in rats and monkeys (Turchi et al. 2005; Winters and Bussey 2005b). Moreover, infusions of scopolamine directly into PRh before the sample phase similarly disrupts object recognition memory (Tang et al. 1997; Warburton et al. 2003; Winters et al. 2006). The amnesic effect of scopolamine in object recognition tasks is most commonly observed when the drug is administered prior to the sample object exposure phase, and administration at other times during the learning and memory process (i.e., post-sample or pre-test) does not consistently disrupt recognition memory (Aigner et al. 1991; Warburton et al. 2003; Winters et al. 2006). These findings suggest a specific role for $\mathrm{ACh}$ in the acquisition of object information, perhaps through a process that amplifies or optimizes cortical processing of sensory stimuli (Hasselmo and Bower 1993; Sarter and Bruno 1997; Hasselmo and McGaughy 2004).

\footnotetext{
${ }^{3}$ Corresponding author.
}

E-mail bdw23@cam.ac.uk; fax 44-0-1223-333564.

Article is online at http://www.learnmem.org/cgi/doi/10.1101//m.634607.
We have recently provided strong support for such a view of cortical ACh (Winters et al. 2006). The findings of our previous study indicated that blockade of muscarinic receptors in PRh can impair or facilitate object recognition memory depending on when this blockade takes place. Specifically, intra-PRh infusions of scopolamine directly before the sample phase disrupted recognition memory for the sample object; infusions of scopolamine within the retention delay or $3 \mathrm{~h}$ prior to the sample phase, however, significantly improved memory performance relative to that observed on saline infusion trials. The particularly poor performance of rats on trials with intra-delay or 3-h-presample saline infusions led us to speculate that the infusion episode might be encouraging the acquisition of retroactively or proactively interfering information. We suggested that a single underlying mechanism-impaired acquisition of new information-could explain these results. That is, the scopolamineinduced facilitation could result from blocking the encoding of interfering information within the retention delay or $3 \mathrm{~h}$ prior to the sample phase in the same way that infusions just before the sample phase impaired encoding of the sample object information. This hypothesis parsimoniously implies that the blockade of information acquisition by scopolamine may disrupt or facilitate memory depending on the task relevance of the information that is blocked.

In our previous study, we posited that saline infusions produced interference by causing a general arousal in the animal, leading to the enhanced encoding of sensory information. The nature of this information, however, is impossible to specify. We therefore designed the current series of experiments to study more explicitly the role of PRh muscarinic receptor-mediated information acquisition in retroactive and proactive object memory interference. To this end, we modified the spontaneous object recognition task to allow the presentation of an interfering object either before the sample phase (proactive interference) or in the retention delay between the sample and choice phases 
(retroactive interference). Experiment 1 used the retroactive interference procedure to test the ability of intra-PRh scopolamine infusions to block the acquisition of retroactively interfering information about the interposed object and thereby preserve recognition memory for the sample object. Experiment 2 assessed the ability of intra-PRh scopolamine infusions to block the acquisition of proactively interfering information from an object presented before the sample phase. Both retroactive and proactive interference effects were observed, and in each case, intra$\mathrm{PRh}$ scopolamine given before exposure to the interfering object reversed the resulting recognition memory impairment. These results are consistent with the proposal that muscarinic cholinergic receptors in PRh are important for the acquisition of object information and that disrupting this acquisition can facilitate recognition memory if the information being blocked would have interfered retroactively or proactively with the to-beremembered object trace.

\section{Results}

Two experiments were run to assess the role of perirhinal muscarinic cholinergic receptors in interference processes affecting object recognition memory. These experiments used modified versions of the standard spontaneous object recognition task for rats, which exploits the natural tendency of rats preferentially to explore novel versus familiar objects. In the present experiments, the spontaneous object recognition procedure was modified so that additional stimuli could be presented either before or after the sample object presentation to study the effects of perirhinal muscarinic receptor blockade on proactive and retroactive memory interference, respectively. In a pilot study (data not shown), a "retroactive interference" phase was introduced in the interval between the sample and choice phases of the task. Rats were exposed on counterbalanced trials to the empty apparatus or the apparatus containing a novel, irrelevant object during the retroactive interference phase. Interposing an object during this intermediate phase retroactively interfered with the sample memory trace, thereby disrupting recognition memory in the choice phase; intact object recognition was observed, however, on trials in which no object was presented during the retroactive interference phase. Experiment 1, therefore, tested the hypothesis that the retroactive interference caused by an interposed object could be prevented by blocking muscarinic receptors within $\mathrm{PRh}$. Rats received counterbalanced intra-PRh infusions of scopolamine or saline $15 \mathrm{~min}$ before the start of the retroactive interference phase of a given trial. Experiment 2 assessed the possibility that pre-sample exposure to an object could interfere proactively with the sample memory trace and that this interference could also be blocked by scopolamine. A "proactive interference" phase was introduced $3 \mathrm{~h}$ before the sample phase. Rats received counterbalanced intra-PRh infusions of scopolamine or saline $15 \mathrm{~min}$ before the start of the proactive interference phase, in which they were exposed to either the empty apparatus or the apparatus containing a novel, irrelevant object. The total memory retention interval between sample and choice phases for both experiments was $3 \mathrm{~h}$. Figure 1 illustrates the basic protocols used for each experiment in the present study.

\section{Experiment 1: Intra-PRh scopolamine prevents the retroactive interference effect caused by an interposed object}

Analysis of the discrimination ratio revealed that, while there was not a significant overall effect of the interposition of an interfering object $\left(F_{(1,7)}=2.8, P>0.1\right)$, there was a significant drug effect $\left(F_{(1,7)}=7.56, P<0.05\right)$, as well as a nearly significant interaction effect between condition and drug $\left(F_{(1,7)}=4.47\right.$,

\section{A. Experiment 1}

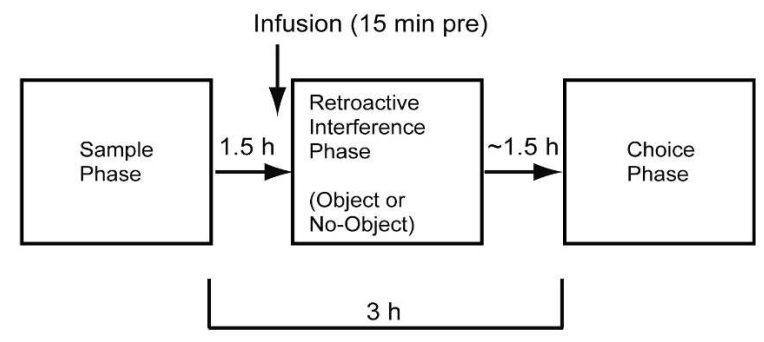

B. Experiment 2

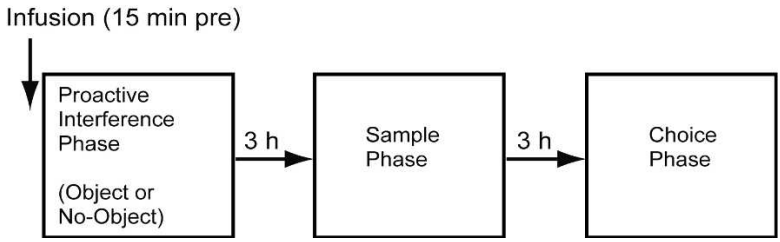

Figure 1. Schematic representation of the procedures used for each of the experiments. The standard spontaneous object recognition paradigm consists of a sample phase, a retention delay, and a choice phase in which the rats are tested for their recognition of the object encountered in the sample phase. The retention delay between the sample and choice phases was $3 \mathrm{~h}$ for both experiments. (A) For Experiment 1, a retroactive interference phase was added within the retention delay, $1.5 \mathrm{~h}$ after the end of the sample phase. Rats were exposed to one of two retroactive interference phase conditions in a within-subjects design. In the object condition, animals were exposed to a novel object in the retroactive interference phase; in the no-object condition, the apparatus was empty when the rats were reintroduced for the retroactive interference phase. In Experiment 1, infusions of saline or scopolamine were administered 15 min before the retroactive interference phase of each trial according to a counterbalanced design. (B) In Experiment 2, a proactive interference phase was added $3 \mathrm{~h}$ before the start of the sample phase. Animals were tested in a within-subjects design with object and no-object conditions in the proactive interference phase. Bilateral intra-PRh infusions of saline or scopolamine were delivered in a counterbalanced fashion, 15 min before the proactive interference phase of each trial.

$P=0.072$ ). Figure 2 shows that rats performed substantially worse on saline trials in the object condition than in the noobject condition, whereas scopolamine infusions prevented this recognition memory impairment. Planned comparisons with paired-samples $t$-tests supported this conclusion, revealing that recognition memory performance on saline and scopolamine trials differed significantly in the object condition $(t=3.44, d f=7$; $P=0.01)$, but not in the no-object condition $(t=0.337, d f=7$; $P>0.7)$.

Analysis of the sample phase duration revealed no significant effects of the interposed object, drug, or the condition by drug interaction (all $F<1$ ); the means $( \pm$ SEM) for separate conditions were as follows: No-object, saline $=76.34 \pm 6.72 \mathrm{sec}$, scopolamine $=78.08 \pm 4.45 \mathrm{sec}$; object, saline $=77.45 \pm 4.2 \mathrm{sec}$, scopolamine $=72.52 \pm 5.08 \mathrm{sec}$. Analysis of the retroactive interference phase duration (time required to accumulate $25 \mathrm{sec}$ of scored exploration; in the no-object condition exploration was scored when the rat was directing attention to one of the three walls in the last third of either arm of the Y-shaped apparatus) indicated significant effects of condition (object or no-object; $\left.F_{(1,7)}=6.09, P<0.05\right)$ and drug $\left(F_{(1,7)}=11.99, P=0.01\right)$, as rats were quicker to achieve the 25 -sec exploration criterion in the no-object compared with the object condition, and following saline compared with scopolamine infusions (means \pm SEM: Noobject, saline $=66.7 \pm 4.15 \mathrm{sec}$, scopolamine $=81.93 \pm 6.77$ sec; object, saline $=70.97 \pm 12.2 \mathrm{sec}$, scopolamine $=111.77 \pm 8.51$ 


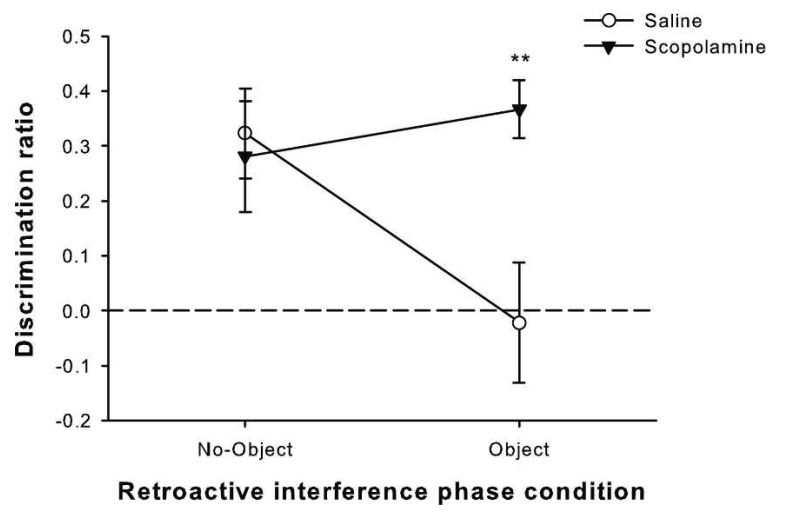

Figure 2. Spontaneous object recognition performance in Experiment 1 on trials in which rats received either saline or scopolamine infusions bilaterally into PRh 15 min before the retroactive interference phase. The retention delay was $3 \mathrm{~h}$. Recognition memory was significantly impaired on trials in which rats received saline infusions prior to exploring an object in the retroactive interference phase (object condition). Scopolamine infusions, however, blocked this memory impairment, maintaining performance at the level observed in the no-object retroactive interference phase condition. Data are presented as average discrimination ratio \pm SEM. $\left.{ }^{* *}\right) P=0.01$, saline versus scopolamine.

sec). The interaction term, however, was not significant $\left(F_{(1,7)}=1.95, P>0.2\right)$, and every rat on every trial explored the interposed objects for $25 \mathrm{sec}$. Absolute object exploration in the first minute of the choice phase was not differentially affected by the retroactive interference phase condition or drug, and the interaction term was also not significant (all $F<1$; means \pm SEM: No-object, saline $=20.88 \pm 2.05 \mathrm{sec}$, scopolamine $=20.24 \pm 2.3$ sec; object, saline $=18.39 \pm 1.72 \mathrm{sec}$, scopolamine $=20.19 \pm 2.57$ sec).

Thus, the results of Experiment 1 suggest that the retroactive interference caused by an interposed object can be blocked by intra-PRh infusions of scopolamine just prior to intermediate object exposure. This effect appears to be the result of the drug blocking the encoding of retroactively interfering object information.

\section{Experiment 2: Pre-sample exposure to an object causes interference that can be blocked by intra-PRh scopolamine}

The discrimination ratio analysis revealed significant effects of the proactive interference phase condition (object or no-object; $\left.F_{(1,9)}=42.3, P<0.001\right)$, as well as the condition by drug interaction $\left(F_{(1,9)}=6.44, P<0.05\right)$; the overall effect of the drug did not reach significance $\left(F_{(1,9)}=3.92, P=0.079\right)$. Figure 3 shows that rats performed substantially worse on saline trials in the object condition than in the no-object condition, and scopolamine infusions prevented this recognition memory impairment. This conclusion was supported by post-hoc tests, which revealed that recognition memory performance on saline and scopolamine trials differed significantly in the object condition $(t=2.86, d f=9$; $P<0.05)$, but not in the no-object condition $(t=0.442, d f=9$; $P>0.6)$.

The sample phase duration analysis yielded no significant effects of the proactive interference phase condition $\left(F_{(1,9)}=1.43, P>0.2\right)$, drug $(F<1)$, or the condition by drug interaction terms $\left(F_{(1,9)}=4.22, P>0.05\right)$; the means \pm SEM for separate conditions were as follows: No-object, saline $=67.06 \pm 5.77 \mathrm{sec}$, scopolamine $=74 \pm 7.23 \mathrm{sec}$; object, saline $=86.7 \pm 10.04 \mathrm{sec}$, scopolamine $=69.98 \pm 5.33 \mathrm{sec}$. Analysis of the proactive interference phase duration indicated a sig- nificant effect of condition $\left(F_{(1,9)}=31.05, P<0.001\right)$, but neither the main effect of drug nor the interaction were significant (both $F<1$; means \pm SEM: No-object, saline $=91.44 \pm 7.6 \mathrm{sec}$, scopolamine $=89.7 \pm 6.88 \mathrm{sec}$; object, saline $=64.78 \pm 10.04 \mathrm{sec}$, scopolamine $=66.13 \pm 4.82 \mathrm{sec}$ ). Every rat on every trial explored the pre-sample objects for $25 \mathrm{sec}$. There were also no significant effects of the proactive interference phase condition $\left(F_{(1,9)}=1.24, P>0.2\right)$, drug $\left(F_{(1,9)}=4.11, P>0.05\right)$, or the interaction $(F<1)$ on total object exploration in the first minute of the choice phase (means \pm SEM: No-object, saline $=12.42 \pm 0.92 \mathrm{sec}$, scopolamine $=13.97 \pm 0.79 \mathrm{sec}$; object, saline $=11.39 \pm 0.83 \mathrm{sec}$, scopolamine $=12.37 \pm 1.1 \mathrm{sec}$ ).

The findings from Experiment 2 therefore suggest that object exposure before the sample phase can adversely affect subsequent recognition memory for the sample object. Antagonism of muscarinic receptors in PRh during the proactive interference phase prevents this impairment, perhaps by disrupting the acquisition of information about the interfering object.

\section{Histology}

All rats included in the behavioral analyses had guide cannulae located bilaterally with injection needle tips terminating in PRh near the border between areas 35 and 36 within cortical layers 2-5 (Burwell 2001). These placements were consistently located between 5.80 and $6.30 \mathrm{~mm}$ posterior to bregma (Fig. 4), the approximate midsection of the rostral-caudal extent of PRh.

\section{Discussion}

The findings of the present study suggest, first, that interference between object memory traces can disrupt object recognition memory both retroactively and proactively. Second, these interference effects critically involve cholinergic modulation via muscarinic receptors within PRh.

In Experiment 1, interposition of object presentations between the sample and choice phases in the spontaneous object recognition task significantly impaired recognition memory. These findings indicate that the acquisition of object information in the retention delay can cause retroactive interference, thereby disrupting recognition memory for the sample object in the choice phase. Infusions of scopolamine into PRh $15 \mathrm{~min}$ before the interposed object exposure blocked the putative ret-

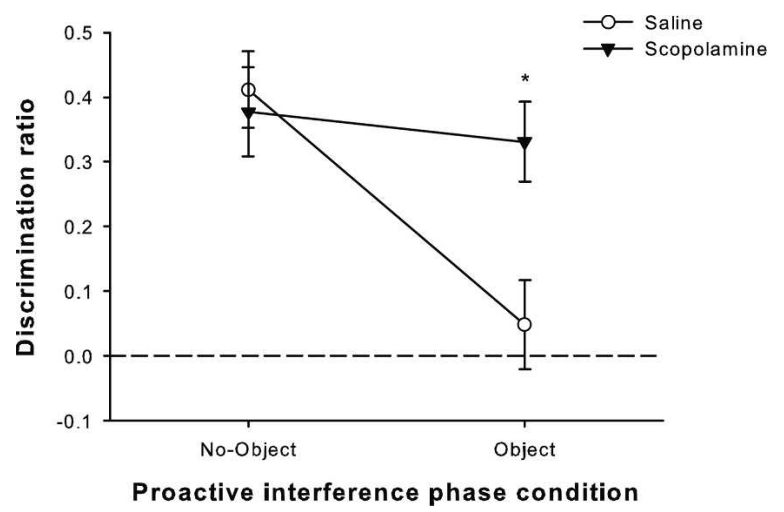

Figure 3. Spontaneous object recognition performance from Experiment 2 on trials in which rats received either saline or scopolamine infusions bilaterally into PRh 15 min before the proactive interference phase. The retention delay was $3 \mathrm{~h}$. Recognition memory was significantly impaired on trials in which rats received saline infusions before pre-sample object exposure (object condition). Conversely, scopolamine infusions prevented this memory impairment, maintaining performance at the level observed in the no-object proactive interference phase condition. Data are presented as average discrimination ratio \pm SEM. $\left({ }^{*}\right) P<0.05$, saline versus scopolamine. 


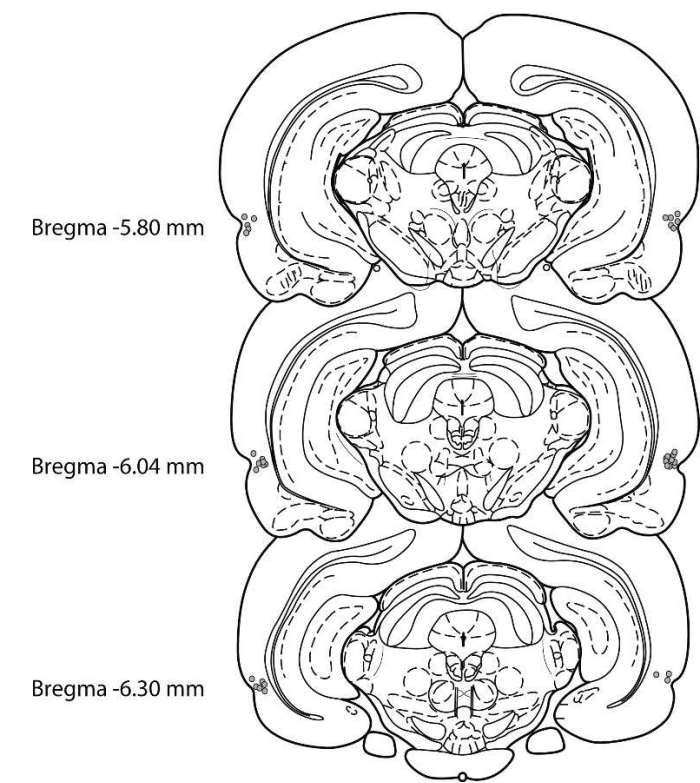

Figure 4. Cannulation of rat PRh. Schematic representation of the infusion cannula tip placements for all rats from Experiments 1 and 2 $(n=19)$. Cannulae were consistently located between 5.80 and $6.30 \mathrm{~mm}$ posterior to bregma. Some cannula tips overlap in the figure. Reprinted with permission from Elsevier (C) 1998, Paxinos and Watson (1998).

roactive interference effect, maintaining recognition memory performance at the same level as in the condition in which rats did not experience an intermediate object presentation. Similarly, the results from Experiment 2 showed that exposure to an object $3 \mathrm{~h}$ before the sample phase proactively interfered with memory for the sample object. As with the retroactive effect in Experiment 1, intra-PRh scopolamine infusions given before the pre-sample object exposure reversed this proactive interference effect; rats given scopolamine behaved as if they had never experienced the interfering item. The results from Experiments 1 and 2 clearly indicate that muscarinic receptors in PRh contribute to the interference effects observed on trials in which an object is presented during the proactive or retroactive interference phase following saline infusions. The fact that these effects can be blocked by intra-PRh infusions of scopolamine just before the proactive or retroactive interference phases suggests that activation of $\mathrm{PRh}$ muscarinic receptors mediates the acquisition of information about the interfering objects. These findings provide further strong support for the notion that PRh and its cholinergic afferents are critical for the acquisition of object information in memory processes.

In a previous study (Winters et al. 2006), the particularly poor performance of rats on trials with intra-delay or 3-h-presample saline infusions led us to suggest that the infusion episode might be encouraging the acquisition of interfering information, perhaps by arousing the animals. We hypothesized that scopolamine infusions protected recognition memory for the sample object by blocking the acquisition of this interfering information at or around the time of the infusion episode. The present study was designed to extend our previous findings (Winters et al. 2006) by analyzing a more explicit case of memory interference, in which the interfering information is known to be of the same type as the to-be-remembered information. Indeed, the results of Experiment 1 indicate that recognition memory can be abolished by interposing a phase of object exploration between the sample and choice phases. Although this result does not confirm the mechanism of interference in the previous study, it does provide support for the hypothesis that memory was impaired by the acquisition of interfering information and was not just a nonspecific effect of saline infusion. Indeed, in the present study, recognition memory was impaired on only the saline infusion trials in which animals were exposed to objects in the proactive or retroactive interference phase. In Winters et al. (2006), the retention delay was $24 \mathrm{~h}$, and it is possible that less salient information than the explicit objects used in the present study could be sufficient to disrupt memory with such a long retention delay.

The present findings are consistent with the established role of PRh in object memory and identification (Buckley and Gaffan 1998; Murray and Bussey 1999; Bussey et al. 2002b). Indeed, the primary implication of the results of the present study is that muscarinic receptors in PRh are critical for the acquisition of object information and that this information can have varying effects on recognition memory depending on its relevance to task performance. If the object information being acquired refers to the to-be-remembered stimulus, then recognition memory will likely be facilitated by muscarinic receptor activation; if, however, the information has the potential to interfere with the target object trace, ACh-mediated acquisition may have a detrimental effect on memory performance. PRh has a clear role in object representation and is involved in aspects of cognition requiring perceptual and/or mnemonic processing of complex object information (Buckley et al. 2001; Bussey et al. 2002a, 2003; Bartko et al. 2007). Consistent with this view, studies have indicated that PRh is crucially involved in the acquisition of object information (Winters and Bussey 2005a,c). Moreover, intra-PRh infusions of scopolamine before the sample phase of object recognition tasks consistently disrupt object recognition memory (Tang et al. 1997; Warburton et al. 2003; Winters et al. 2006), indicating an important role for cholinergic modulation in PRhmediated object information acquisition. Such a role is consistent with electrophysiological studies indicating that cholinergic activation can fine-tune the receptive fields of cortical pyramidal cells (Krnjevic and Phillis 1963; Sillito and Kemp 1983; Murphy and Sillito 1991; Rasmusson 2000) and human neuroimaging findings, which suggest that cortical ACh can enhance perceptual processing and encoding in visual memory tasks (Furey et al. 1997, 2000; Schon et al. 2005). Moreover, ACh has been implicated in synaptic plasticity processes within PRh (Massey et al. 2001; Warburton et al. 2003), and this function is probably related to the role of ACh in enhancing cortical information processing (Sarter and Bruno 1997; Rasmusson 2000). The present interpretation is consistent with the view that increased cortical ACh levels encourage encoding by enhancing afferent input and suppressing excitatory intrinsic circuitry (Hasselmo and Bower 1993; Linster et al. 2003; Hasselmo and McGaughy 2004). IntraPRh infusions of scopolamine administered before the acquisition of new object information may disrupt such a process, reducing the selectivity of cortical responsiveness to incoming sensory information about the object being explored. Such a mechanism would explain both the detrimental effects of presample scopolamine and the positive effects of scopolamine infusions at other phases within an object recognition trial; in both cases, the acquisition of object information is prevented, but the consequences of this disruption depend on the task relevance of the objects being presented.

The results of the present study provide further support for the view that PRh and its cholinergic afferents are crucially involved in the acquisition of object information for recognition memory processes. Moreover, these findings suggest that the neuromodulatory role of ACh in object memory acquisition can influence recognition memory directly via an enhancement of sample object encoding, or indirectly via a similar strengthening 
of interfering information acquisition. Viewed in this way, the seemingly paradoxical facilitation of object recognition memory by intra-PRh scopolamine infusions in the present study can be explained by the same underlying mechanism through which intra-PRh scopolamine impairs memory when administered before the acquisition of task-relevant information.

\section{Materials and Methods}

\section{Animals}

The subjects were 19 adult male Lister Hooded rats (Harlan Olac), weighing 270-320 g prior to surgery and housed in pairs in a room with a 12-h light/12-h dark cycle (lights on at 7:00 P.M.). Different batches of rats were used for each experiment (Experiment 1, $n=9$; Experiment 2, $n=10$ ). All behavioral testing was conducted during the dark phase of the cycle. During testing, rats were fed $\sim 15 \mathrm{~g}$ of laboratory chow following daily behavioral sessions to maintain weights at $85 \%-90 \%$ of free-feeding body weight. Water was available ad libitum throughout the experiment. All experimentation was conducted in accordance with the UK Animals (Scientific Procedures) Act, 1986.

\section{Surgery}

For each experiment, all rats were implanted bilaterally with 22gauge indwelling guide cannulae according to the following procedure. Prior to surgery, all animals were deeply anaesthetized by intraperitoneal (i.p.) injection $(60 \mathrm{mg} / \mathrm{kg}$ ) of sodium pentobarbital (Sagatal; Rhône Mérieux) and placed in a stereotaxic frame (David Kopf Instruments) with the incisor bar set at $-3.2 \mathrm{~mm}$. The scalp was cut and retracted to expose the skull. Holes were drilled and the guide cannulae implanted according to the following coordinates, measured relative to the skull at bregma (Paxinos and Watson 1998): anteroposterior $-5.5 \mathrm{~mm}$, lateral $\pm 6.6 \mathrm{~mm}$, dorsoventral $-6.5 \mathrm{~mm}$. The cannulae were secured to the skull using four jeweler screws and dental acrylic. Obturators cut to extend $1.1 \mathrm{~mm}$ beyond the tip of the guide cannulae and with an outer diameter of $0.36 \mathrm{~mm}$ were inserted into the guides and remained there except during infusions. At the completion of each surgery, the skin was sutured, and an antibiotic powder (Acramide; Dales Pharmaceuticals) was applied. Animals were allowed to recover for at least $7 \mathrm{~d}$ prior to the beginning of behavioral testing.

\section{Spontaneous object recognition}

Spontaneous object recognition was tested in a Y-shaped apparatus, as described previously (Winters et al. 2004; Forwood et al. 2005). Briefly, the Y-shaped apparatus had high, homogeneous white walls constructed from Foamalux (Brett Martin) to prevent the rat from looking out into the room and, thereby, to maximize attention to the object stimuli. The apparatus was raised $30 \mathrm{~cm}$ from the floor with walls $40 \mathrm{~cm}$ high. Each arm was $27 \mathrm{~cm}$ in length and $10 \mathrm{~cm}$ wide. The start arm contained a guillotine door $18 \mathrm{~cm}$ from the rear of the arm. This provided a start box area within which the rat could be confined at the start of the sample and choice phases of a given trial. The floor and walls of the apparatus were wiped down with a dry paper towel between rats, but otherwise were not cleaned during the experiment. A video camera was mounted above the apparatus to record all trials. Triplicate copies were obtained of the objects, which were made of glass, plastic, or metal. For any given trial, the objects in a pair were composed of the same material so that they could not readily be distinguished by olfactory cues. The height of the objects ranged from 5 to $20 \mathrm{~cm}$, and all objects were affixed to the floor of the apparatus with Blu Tack (Bostik) to prevent them from being displaced during a trial. As far as could be determined, the objects had no natural significance for the rats, and they had never been associated with a reinforcer.

All rats were habituated in two consecutive daily sessions in which they were allowed to explore the empty Y-shaped appara- tus for $5 \mathrm{~min}$. Prior to being placed in the apparatus, rats experienced a mock infusion procedure, as described below. Following the mock infusion, the rats were taken from the preparation area to the testing room and placed in the start box; the guillotine door was then opened to allow the rat to explore the main area of the apparatus. When the rat exited the start box, the guillotine door was lowered to prevent re-entry into this area of the apparatus. The experimenter did not begin timing the session until after the rat exited the start box.

Testing began $24 \mathrm{~h}$ after the second habituation session. At the start of each day, rats were transported to holding cages in a room adjacent to the main testing area. The rats remained in these holding cages throughout the day, except when they were being tested in the Y-shaped apparatus or receiving infusions. When all behavioral testing for that day was complete, the rats were returned to their home cages and fed. Rats were given a series of test trials, with a minimum interval of $24 \mathrm{~h}$ between trials. A different object pair was used for each trial for a given animal, and the order of exposure to object pairs as well as the designated sample and novel objects for each pair were counterbalanced. The time spent exploring objects was assessed from video recordings of the sample and choice phases. Data were collected by scoring exploratory bouts using a personal computer running a program written in Visual Basic 6.0. All trials were run with the experimenter blind to the drug treatment conditions.

Each trial consisted of three phases: the standard sample and choice phases, plus an additional interference phase. This additional phase provided the critical modification of the paradigm for the present study by allowing the introduction of explicit object interference into the structure of trials. The nature of the additional phase changed depending on the experiment and is, therefore, described in detail under the specific experiment headings below. In the sample phase, two identical objects (A1 and A2) were placed in the Y-shaped apparatus, one at the end of each exploration arm. The rat was placed in the start box with the guillotine door lowered. The guillotine door was then raised to allow the rat into the exploration area of the maze. When the rat exited the start box, the guillotine door was lowered to prevent re-entry, and the sample phase began. The time spent exploring the two objects was scored by an experimenter viewing the rat on a video screen. The cumulative duration of exploratory bouts, the beginning and end of which were indicated by pressing a given key on the computer keyboard, was calculated by the computer program. Exploration of an object was defined as directing the nose to the object at a distance of $<2 \mathrm{~cm}$ and/or touching it with the nose. The sample phase ended when the rat had explored the identical objects for a total of $25 \mathrm{sec}$.

At the end of the sample phase, the rat was removed from the Y-shaped apparatus and, depending on the experiment, was either placed back into its holding cage until the retroactive interference phase (Experiment 1, see below) or for the duration of the retention delay (Experiment 2). The retention delay between the sample and choice phases was $3 \mathrm{~h}$ in both experiments. After the delay, the rat was placed back in the start box of the Y-shaped apparatus and released into the exploration area for the choice phase. In the choice phase, the Y-shaped apparatus contained an identical copy of the sample (familiar) object (A3) in one arm and a new object (B) in the other. The exploration arms in which the choice objects were placed were counterbalanced between rats and across trials. The rat was allowed to explore the objects for 2 min, at the end of which it was removed and returned to its home cage. The time spent exploring the novel and familiar objects was recorded for the 2 min of the choice phase, but attention was focused on the first minute, during which object discrimination is typically greatest. We calculated a discrimination ratio, the proportion of total exploration time spent exploring the novel object (i.e., the difference in time spent exploring the novel and familiar objects divided by the total time spent exploring the objects), for the first minute of the choice phase on each object recognition trial. This measure takes into account individual differences in the total amount of exploration time.

Figure 1 illustrates the basic protocols used for the experiments described below. 
Experiment 1: Can intra-PRh scopolamine block the retroactive interfering effects of an interposed object on recognition memory?

Experiment 1 was conducted to test the hypothesis that scopolamine could prevent the detrimental effect on object recognition of interposing an irrelevant object between the sample and choice phases. Thus, in this experiment, the additional phase within a trial, called the "retroactive interference phase," was conducted during the retention interval between the sample and choice phases. On all trials, 75 min after the end of the sample phase, rats received intra-PRh infusions of saline or scopolamine. Fifteen minutes later, animals were placed into the Y-shaped apparatus under one of two retroactive interference phase conditions. In the "no-object" condition, the apparatus was empty; "exploration" in this condition was scored when the rat was directing attention to one of the three walls (i.e., directing the nose at the wall at a distance of $<2 \mathrm{~cm}$ and/or touching the wall with the nose) within the last third of either arm of the Y-shaped apparatus; this region is equivalent to the area occupied by objects when they are placed in the apparatus. Rats remained in the apparatus until they had accumulated $25 \mathrm{sec}$ of total exploration between the left and right arms or until $3 \mathrm{~min}$ had passed, whichever came first. In the "object" condition, the rats were exposed to a novel pair of identical objects, one at the end of each arm of the Y-shaped apparatus. Rats remained in the apparatus until they had accumulated $25 \mathrm{sec}$ of total object exploration or until $3 \mathrm{~min}$ had passed, whichever came first; object exploration was scored as usual in this condition. We predicted that the object condition would produce retroactive interference with the sample trace, thereby disrupting subsequent recognition memory performance in the choice phase, which took place $\sim 90$ min after the end of the retroactive interference phase. The sample-to-choice retention interval was $3 \mathrm{~h}$ in total. The object sets used for the object condition in the retroactive interference phase were different from those used for the sample and choice phases. Both drug (saline or scopolamine) and retroactive interference phase (object or no-object) variables were tested in a fully within-subjects design such that all rats received eight total trials, two trials with each condition combination: saline-object, saline-no-object, scopolamine-object, and scopolamine-noobject. The orders of trial types, exposure to retroactive interference phase object sets, and drug infusions were counterbalanced across rats.

\section{Experiment 2: Can intra-PRh scopolamine block the proactive interfering effects of pre-sample object exposure on recognition memory?}

Experiment 2 studied the effects of pre-sample object exposure on recognition memory for the sample object and the ability of scopolamine to block the predicted proactive interference effects. In this experiment, the additional phase within a trial, called the "proactive interference phase," occurred $3 \mathrm{~h}$ before the sample phase. In all other aspects, the proactive interference phase in Experiment 2 was identical to the retroactive interference phase in Experiment 1. Rats received infusions of either saline or scopolamine $15 \mathrm{~min}$ before being placed into the Y-shaped apparatus for the proactive interference phase, which was conducted with both object and no-object conditions. The proactive interference phase continued until the rats accumulated $25 \mathrm{sec}$ of exploration or $3 \mathrm{~min}$ had passed, whichever came first. Exploration in each condition was scored in the same way as in Experiment 1 . At the end of the proactive interference phase, the rats were placed back into their holding cages for $3 \mathrm{~h}$ until the sample phase. The trial then proceeded as usual, with a 3-h retention delay between sample and choice phases. Both drug (saline or scopolamine) and proactive interference phase (object or noobject) variables were tested in a fully within-subjects design such that all rats received eight total trials, two trials with each condition combination: saline-object, saline-no-object, scopolamine-object, and scopolamine-no-object. The object sets used for the object condition in the proactive interference phase were different from those used for the sample and choice phases. The orders of trial types, presentation of proactive interference phase object sets, and drug infusions were counterbalanced across rats.

\section{Infusion procedure}

For both experiments, rats received bilateral infusions of either physiological saline $(0.9 \%$ sodium chloride at $\mathrm{pH} 7.0$; Aquapharm, Animalcare Limited) or scopolamine hydrobromide trihydrate $(10 \mathrm{mg} / \mathrm{mL}$ in physiological saline; Sigma) on a given trial, 15 min prior to the start of the retroactive (Experiment 1) or proactive (Experiment 2) interference phase. For all conditions, the basic procedure was as follows. All infusions took place in a preparation room separate from the behavioral testing area. Animals were gently restrained by the experimenter throughout the infusion process. The obturators were removed, and 28-gauge infusion cannulae, which were cut to extend $1 \mathrm{~mm}$ beyond the tip of the guides, were inserted into the guides. Bilateral infusions were conducted simultaneously using two 1- $\mu \mathrm{L}$ Hamilton syringes, which were connected to the infusion cannulae by propylene tubing. The syringes were driven by a Harvard Apparatus precision syringe pump, which delivered $1 \mu \mathrm{L}$ to each hemisphere over $2 \mathrm{~min}$. The infusion cannulae were left in place for an additional $1.5 \mathrm{~min}$ to allow for diffusion of the infusate. The infusion cannulae were then removed, and the obturators replaced before the next phase of the procedure (see above for specific experiment parameters). For both experiments, in each of the two habituation sessions prior to the beginning of behavioral testing, rats experienced a mock infusion identical in all aspects to the procedure described above, except that the injection cannulae contained no liquid. This was done to habituate the animals to the infusion procedure.

\section{Histology}

Following behavioral testing, rats were anaesthetized by i.p. injection of $2 \mathrm{~mL}$ of Dolethal (Rhône Mérieux) and perfused transcardially with $100 \mathrm{~mL}$ of phosphate buffered saline (PBS at $\mathrm{pH}$ 7.4), followed by $250 \mathrm{~mL}$ of $4 \%$ paraformaldehyde (PFA at $\mathrm{pH}$ 7.4). The brains were removed, postfixed in $4 \%$ PFA for $24 \mathrm{~h}$ at $4^{\circ} \mathrm{C}$, and then immersed in $25 \%$ sucrose in PBS until they sank. Coronal sections $(60 \mu \mathrm{m})$ were cut on a freezing microtome through the extent of PRh, and every fifth section was mounted on a gelatin-coated glass slide and stained with cresyl violet. Slides were examined under a light microscope to verify the cannula placements, and approximate cannula tip locations were mapped onto sections by comparing visually with slides from Paxinos and Watson (1998).

\section{Data analysis}

Group means of three measures taken from object recognition testing (duration of the sample phase, object exploration time in the first minute of the choice phase, and the discrimination ratio from the first minute of the choice phase) were analyzed for each experiment. The duration of the retroactive (Experiment 1) or proactive (Experiment 2) interference phase was also analyzed. Means from all measures were submitted to two-way analyses of variance (ANOVA) with repeated measures for Experiments 1 and 2 (retroactive or proactive interference phase condition by drug). Paired-samples $t$-tests were used for post hoc analyses of withinsubjects effects. All statistical analyses were conducted with a significance level of $\alpha=0.05$.

\section{Acknowledgments}

This work was supported by a Biotechnology and Biological Sciences Research Council Grant to B.D.W., L.M.S., and T.J.B. S.J.B. was additionally supported by a Ruth L. Kirchstein Predoctoral Fellowship from the National Institute of Mental Health.

\section{References}

Aigner, T.G. and Mishkin, M. 1986. The effects of physostigmine and scopolamine on recognition memory in monkeys. Behav. Neural Biol. 45: 81-87. 
Aigner, T.G., Walker, D.L., and Mishkin, M. 1991. Comparison of the effects of scopolamine administered before and after acquisition in a test of visual recognition memory in monkeys. Behav. Neural Biol. 55: $61-67$.

Bartko, S.J., Winters, B.D., Cowell, R.A., Saksida, L.M., and Bussey, T.J. 2007. Perceptual functions of perirhinal cortex in rats: Zero-delay object recognition and simultaneous oddity discriminations. $J$. Neurosci. 27: 2548-2559.

Bartus, R.T. 2000. On neurodegenerative diseases, models, and treatment strategies: Lessons learned and lessons forgotten a generation following the cholinergic hypothesis. Exp. Neurol. 163: 495-529.

Buckley, M.J. and Gaffan, D. 1998. Perirhinal cortex ablation impairs visual object identification. J. Neurosci. 18: 2268-2275.

Buckley, M.J., Booth, M.C., Rolls, E.T., and Gaffan, D. 2001. Selective perceptual impairments after perirhinal cortex ablation. J. Neurosci. 21: 9824-9836.

Buffalo, E.A., Reber, P.J., and Squire, L.R. 1998. The human perirhinal cortex and recognition memory. Hippocampus 8: 330-339.

Burwell, R.D. 2001. Borders and cytoarchitecture of the perirhinal and postrhinal cortices in the rat. J. Comp. Neurol. 437: 17-41.

Bussey, T.J., Saksida, L.M., and Murray, E.A. 2002a. Perirhinal cortex resolves feature ambiguity in complex visual discriminations. Eur. J. Neurosci. 15: 365-374.

Bussey, T.J., Saksida, L.M., and Murray, E.A. 2002b. The role of perirhinal cortex in memory and perception: Conjunctive representations for object identification. In The parahippocampal region: Organization and role in cognitive functions (eds. M.P. Witter and F.G. Wouterlood), pp. 239-254. Oxford University Press, Oxford.

Bussey, T.J., Saksida, L.M., and Murray, E.A. 2003. Impairments in visual discrimination after perirhinal cortex lesions: Testing 'declarative' vs. 'perceptual-mnemonic' views of perirhinal cortex function. Eur. J. Neurosci. 17: 649-660.

Dodart, J.C., Mathis, C., and Ungerer, A. 1997. Scopolamine-induced deficits in a two-trial object recognition task in mice. Neuroreport 8: $1173-1178$.

Ennaceur, A. and Meliani, K. 1992. Effects of physostigmine and scopolamine on rats' performances in object-recognition and radial-maze tests. Psychopharmacology 109: 321-330.

Everitt, B.J. and Robbins, T.W. 1997. Central cholinergic systems and cognition. Annu. Rev. Psychol. 48: 649-684.

Forwood, S.E., Winters, B.D., and Bussey, T.J. 2005. Hippocampal lesions that abolish spatial maze performance spare object recognition memory at delays of up to 48 hours. Hippocampus 15: 347-355.

Furey, M.L., Pietrini, P., Haxby, J.V., Alexander, G.E., Lee, H.C., VanMeter, J., Grady, C.L., Shetty, U., Rapoport, S.I., Schapiro, M.B., et al. 1997. Cholinergic stimulation alters performance and task-specific regional cerebral blood flow during working memory. Proc. Natl. Acad. Sci. 94: 6512-6516.

Furey, M.L., Pietrini, P., and Haxby, J.V. 2000. Cholinergic enhancement and increased selectivity of perceptual processing during working memory. Science 290: 2315-2319.

Hasselmo, M.E. and Bower, J.M. 1993. Acetylcholine and memory. Trends Neurosci. 16: 218-222.

Hasselmo, M.E. and McGaughy, J. 2004. High acetylcholine levels set circuit dynamics for attention and encoding and low acetylcholine levels set dynamics for consolidation. Prog. Brain Res. 145: 207-231.

Huston, A.E. and Aggleton, J.P. 1987. The effects of cholinergic drugs upon recognition memory in rats. Q. J. Exp. Psychol. B 39: 297-314.

Krnjevic, K. and Phillis, J.W. 1963. Acetylcholine-sensitive cells in the cerebral cortex. J. Physiol. 166: 296-327.
Linster, C., Maloney, M., Patil, M., and Hasselmo, M.E. 2003. Enhanced cholinergic suppression of previously strengthened synapses enables the formation of self-organized representations in olfactory cortex. Neurobiol. Learn. Mem. 80: 302-314.

Massey, P.V., Bhabra, G., Cho, K., Brown, M.W., and Bashir, Z.I. 2001. Activation of muscarinic receptors induces protein synthesisdependent long-lasting depression in the perirhinal cortex. Eur. J. Neurosci. 14: 145-152.

Meunier, M., Bachevalier, J., Mishkin, M., and Murray, E.A. 1993. Effects on visual recognition of combined and separate ablations of the entorhinal and perirhinal cortex in rhesus monkeys. J. Neurosci. 13: 5418-5432.

Murphy, P.C. and Sillito, A.M. 1991. Cholinergic enhancement of direction selectivity in the visual cortex of the cat. Neuroscience 40: $13-20$.

Murray, E.A. and Bussey, T.J. 1999. Perceptual-mnemonic functions of the perirhinal cortex. Trends Cogn. Sci. 3: 142-151.

Paxinos, G. and Watson, C. 1998. The rat brain in stereotaxic coordinates. Academic Press, London.

Rasmusson, D.D. 2000. The role of acetylcholine in cortical synaptic plasticity. Behav. Brain Res. 115: 205-218.

Sarter, M. and Bruno, J.P. 1997. Cognitive functions of cortical acetylcholine: Toward a unifying hypothesis. Brain Res. Brain Res. Rev. 23: 28-46.

Schon, K., Atri, A., Hasselmo, M.E., Tricarico, M.D., LoPresti, M.L., and Stern, C.E. 2005. Scopolamine reduces persistent activity related to long-term encoding in the parahippocampal gyrus during delayed matching in humans. J. Neurosci. 25: 9112-9123.

Sillito, A.M. and Kemp, J.A. 1983. Cholinergic modulation of the functional organization of the cat visual cortex. Brain Res. 289: $143-155$.

Tang, Y., Mishkin, M., and Aigner, T.G. 1997. Effects of muscarinic blockade in perirhinal cortex during visual recognition. Proc. Natl. Acad. Sci. 94: 12667-12669.

Turchi, J., Saunders, R.C., and Mishkin, M. 2005. Effects of cholinergic deafferentation of the rhinal cortex on visual recognition memory in monkeys. Proc. Natl. Acad. Sci. 102: 2158-2161.

Warburton, E.C., Koder, T., Cho, K., Massey, P.V., Duguid, G., Barker, G.R., Aggleton, J.P., Bashir, Z.I., and Brown, M.W. 2003. Cholinergic neurotransmission is essential for perirhinal cortical plasticity and recognition memory. Neuron 38: 987-996.

Winters, B.D. and Bussey, T.J. 2005a. Glutamate receptors in perirhinal cortex mediate encoding, retrieval, and consolidation of object recognition memory. J. Neurosci. 25: 4243-4251.

Winters, B.D. and Bussey, T.J. 2005b. Removal of cholinergic input to perirhinal cortex disrupts object recognition but not spatial working memory in the rat. Eur. J. Neurosci. 21: 2263-2270.

Winters, B.D. and Bussey, T.J. 2005c. Transient inactivation of perirhinal cortex disrupts encoding, retrieval, and consolidation of object recognition memory. J. Neurosci. 25: 52-61.

Winters, B.D., Forwood, S.E., Cowell, R.A., Saksida, L.M., and Bussey, T.J. 2004. Double dissociation between the effects of peri-postrhinal cortex and hippocampal lesions on tests of object recognition and spatial memory: Heterogeneity of function within the temporal lobe. J. Neurosci. 24: 5901-5908.

Winters, B.D., Saksida, L.M., and Bussey, T.J. 2006. Paradoxical facilitation of object recognition memory after infusion of scopolamine into perirhinal cortex: Implications for cholinergic system function. J. Neurosci. 26: 9520-9529.

Received April 23, 2007; accepted in revised form July 20, 2007. 


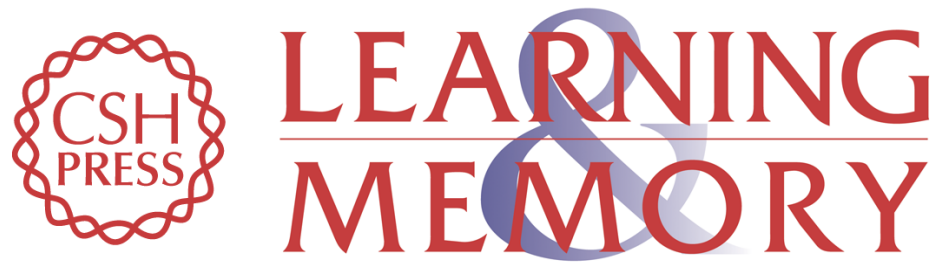

\section{Scopolamine infused into perirhinal cortex improves object recognition memory by blocking the acquisition of interfering object information}

Boyer D. Winters, Susan J. Bartko, Lisa M. Saksida, et al.

Learn. Mem. 2007, 14:

Access the most recent version at doi:10.1101//m.634607

References This article cites 37 articles, 13 of which can be accessed free at: http://learnmem.cshlp.org/content/14/9/590.full.html\#ref-list-1

License

Email Alerting Receive free email alerts when new articles cite this article - sign up in the box at the Service top right corner of the article or click here. 